\title{
DIAGNÓSTICO PARA PLANEJAR AÇÕES DE PROMOÇÃO DA ALIMENTAÇÃO SAUDÁVEL NA ESCOLA*
}

\author{
Ana Eliza Port Lourenço ${ }^{1}$ \\ https://orcid.org/0000-0002-9619-8052 \\ Ana Laura Nunes Machado 2 \\ https://orcid.org/0000-0003-2149-5455 \\ Luana Silva Monteiro ${ }^{3}$ \\ https://orcid.org/0000-0003-3599-6947
}

Priscila Vieira Pontes 4

https://orcid.org/0000-0003-3914-9307

Naiara Sperandio 5

http://orcid.org/0000-0002-9015-3849

Recebido em: 31/07/2021.

Publicado em: 30/09/2021.

\section{RESUMO}

Este artigo apresenta o resultado de um diagnóstico situacional para planejar ações de Promoção da Alimentação Adequada e Saudável (PAAS) em escolas públicas de Macaé, RJ. Trata-se de uma etapa de um estudo de intervenção, ocorrido em 2018/2019, no âmbito das atividades do Núcleo de Estudos em Saúde e Nutrição na Escola (NESANE), referente à identificação de pontos críticos para direcionamento de ações de PAAS em escolas. A metodologia empregada foi descritiva e exploratória. Foram feitas revisão bibliográfica e duas oficinas para discutir a bibliografia analisada e identificar pontos críticos para propor ações. A revisão incluiu apenas publicações do NESANE no período de 2014 a 2019. Foram identificados oito pontos críticos, que se classificaram em cinco temáticas pertinentes à PAAS: perfil antropométrico, perfil alimentar, ambiente alimentar, educação alimentar e nutricional, e comunicação em saúde. A partir desses pontos, foram sugeridos objetivos norteadores de ações, tais como favorecer o consumo de água pelos estudantes e retirar cantinas não saudáveis do interior das escolas. Para cada objetivo norteador, foram apontados exemplos de ações de PAAS possíveis de serem realizadas nas dimensões de incentivo, apoio e proteção. O diagnóstico produzido fornece respaldo para que diferentes atores sociais possam, dentro de seu nível de gerência, desenvolver

\footnotetext{
${ }^{1}$ Núcleo de Estudos em Saúde e Nutrição na Escola - NESANE, Campus UFRJ Macaé.

${ }^{2}$ Núcleo de Estudos em Saúde e Nutrição na Escola - NESANE, Campus UFRJ Macaé.

${ }^{3}$ Núcleo de Estudos em Saúde e Nutrição na Escola - NESANE, Campus UFRJ Macaé.

${ }^{4}$ Núcleo de Estudos em Saúde e Nutrição na Escola - NESANE, Campus UFRJ Macaé.

${ }^{5}$ Núcleo de Estudos em Saúde e Nutrição na Escola - NESANE, Campus UFRJ Macaé.
} 
ações de PAAS nas escolas de Macaé. Ao apontar pontos críticos em nível local, este diagnóstico agrega concretude aos problemas, promove reflexão entre os envolvidos e pode contribuir para a materialização de ações, favorecendo a transformação social.

Palavras-chave: serviços de saúde escolar; alimentação escolar; saúde pública; pesquisa sobre serviços de saúde; programas e políticas de nutrição e alimentação.

\title{
DIAGNOSIS TO PLAN ACTIONS TO PROMOTE HEALTHY EATING IN SCHOOLS
}

\begin{abstract}
This article presents the result of a situational diagnosis to plan actions of Promotion of Adequate and Healthy Eating (PAHE) in public schools of Macaé - a Brazilian municipality in the northern region of the state of Rio de Janeiro. This is one of the phases of an intervention study, carried out in 2018/2019, within the scope of the activities of the "Núcleo de Estudos em Saúde e Nutrição na Escola" (NESANE), referent to the identification of critical points to orient PAHE actions in schools. The methods were descriptive and exploratory. We did literature review and two workshops to discuss this literature and identify critical points to propose actions. The review included only NESANE's publications from 2014 to 2019. We identified eight critical points, which were classified into five themes relevant to PAHE: anthropometric profile, eating profile, food environment, food and nutrition education, and health communication. Based on these points, we suggested action guiding objectives, such as to encourage water intake by students and remove unhealthy canteens from schools. For each guiding objective, we gave examples of PAHE actions that could be caried out in the dimensions of encouragement, support, and protection. This diagnosis provides support for different social actors so they can, within their management level, develop PAHE actions in Macaé schools. By pointing out critical points at the local level, this diagnosis adds concreteness to problems, promotes reflection among those involved, and can contribute to the materialization of actions, favoring social transformation.
\end{abstract}

Keywords: school health services; school feeding; public health; health services research; nutrition programs and policies.

\section{DIAGNÓSTICO PARA PLANIFICAR ACCIONES DE PROMOCIÓN DE LA ALIMENTACIÓN SALUDABLE EN LA ESCUELA}

\section{RESUMEN}

Este artículo presenta el resultado de un diagnóstico situacional para planificar acciones de Promoción de la Alimentación Adecuada y Saludable (PAAS) en escuelas públicas de Macaé - un municipio brasileño de la región norte del estado de Río de Janeiro. Se trata de un paso en un estudio de intervención, realizado en 2018/2019, en el ámbito de las actividades del "Núcleo de Estudos em Saúde e Nutrição na Escola" (NESANE), en cuanto a la identificación de puntos críticos para orientar acciones del PAAS en las escuelas. La metodología utilizada fue descriptiva y exploratoria. Se realizó una revisión bibliográfica y dos talleres para discutir la bibliografía analizada y identificar puntos críticos para proponer acciones. La revisión incluyó solo publicaciones de NESANE de 2014 a 2019. Se identificaron ocho puntos críticos, que se 
clasificaron en cinco temas relevantes para el PAAS: perfil antropométrico, perfil alimentario, ambiente alimentario, educación alimentaria y nutricional, y comunicación en salud. A partir de estos puntos, se sugirieron objetivos rectores de acciones, como fomentar el consumo de agua por parte de los estudiantes y retirar los comedores no saludables del interior de las escuelas. Para cada objetivo rector se señalaron ejemplos de acciones del PAAS que se podrían realizar en las dimensiones de incentivo, apoyo y protección. El diagnóstico elaborado brinda apoyo a los diferentes actores sociales para que puedan, dentro de su nivel de gestión, desarrollar acciones del PAAS en las escuelas de Macaé. Al señalar puntos críticos a nivel local, este diagnóstico agrega concreción a los problemas, promueve la reflexión entre los involucrados y puede contribuir a la materialización de acciones, favoreciendo la transformación social.

Palabras Clave: servicios de salud escolar; alimentación escolar; salud pública; investigación sobre servicios de salud; programas y políticas de nutrición y alimentación.

\section{INTRODUÇÃO}

A extensão universitária constitui um processo interdisciplinar, educativo, cultural e científico de interação com a sociedade (FORPROEX, 2012). Especialmente em campi de interiorização, como o caso do campus da Universidade Federal do Rio de Janeiro em Macaé (Campus UFRJ-Macaé), atividades de extensão tendem a ser frequentes, uma vez que a interiorização tem por princípio ampliar o envolvimento das universidades com as redes locais de serviços e com a comunidade (BRASIL, 2015). Ressalta-se que, desde sua implantação em 2007, o Campus UFRJ-Macaé busca interagir com a sociedade por meio de projetos de extensão diversos, em especial aqueles voltados para a promoção de saúde (LOURENÇO; BERGOLD, 2019).

O vínculo academia-comunidade resultante desses projetos favorece o desenvolvimento de intervenções contextualizadas localmente e, por conseguinte, com maior potencial transformador (FORPROEX, 2012). Por meio de relação interdependente e indissociável com o ensino e a pesquisa, a extensão cria um campo fértil para a formação universitária e para pesquisas voltadas para a ação. Sendo assim, a extensão tem amplo potencial de contribuição para o desenvolvimento regional.

Dentre os cenários para materialização da prática extensionista no campo da saúde, destacase a escola, sobretudo por se tratar de um ambiente que promove a formação de hábitos e a construção de valores, conhecimentos e saberes de várias naturezas. A escola é um espaço- chave para ações de Promoção da Alimentação Adequada e Saudável (PAAS), bem como de outras intervenções que visam o controle ou a prevenção de problemas de saúde e nutrição 
(BRASIL, 2009; POLICY BRIEF, 2021). Cabe comentar que o consumo alimentar dos brasileiros, incluindo os escolares, caracteriza-se por elevada frequência de produtos ultraprocessados, e baixa participação de verduras, legumes e frutas - perfil francamente associado com a ocorrência de obesidade e outras doenças crônicas não transmissíveis (IBGE, 2016; IBGE, 2020).

Esforços internacionais e nacionais têm ocorrido com vistas a aprimorar a alimentação, a saúde e a qualidade de vida nas sociedades (WORLD HEALTH ORGANIZATION, 2004; BRASIL, 2011). É desejável que o município, seja Macaé ou outros, enquanto ente federativo mais próximo dos cidadãos, promova ações locais alinhadas com os planejamentos em esfera federal. Para isso, diagnósticos situacionais são fundamentais, pois permitem identificar problemas específicos e direcionar de forma assertiva políticas e ações nas localidades. Cabe citar que a necessidade de olhar para Macaé e refletir sobre sua história e demandas sociais já foi apontada anteriormente (SILVA; CARVALHO, 2019).

Desde 2018, a temática da Educação Alimentar e Nutricional passou a integrar o currículo de todos os segmentos da educação básica no Brasil (BRASIL, 2018). Vive-se, portanto, um momento oportuno para a PAAS nas escolas, pois essas vêm buscando se adequar à nova legislação, o que pode favorecer a reflexão da comunidade escolar sobre o tema. É também fundamental neste momento apoiar os professores e a direção das escolas para facilitar a concretização de ações de PAAS no ambiente escolar.

A partir de um diagnóstico em nível local, uma gama de ações de PAAS podem ser realizadas no espaço escolar. Essas podem ter como objetivo informar e sensibilizar os participantes, facilitar o acesso a alimentos saudáveis, fortalecer regulamentações sobre alimentação saudável, dentre outras possibilidades (LOURENÇO; PONTES, 2019a). Conforme cada realidade é possível planejar atividades presenciais ou à distância, voltadas para diferentes integrantes da comunidade escolar.

Nessa direção, o presente artigo apresenta o resultado de um diagnóstico situacional para fins de desenvolvimento de ações de PAAS em escolas públicas de Macaé. Sua organização é composta de três seções, além desta introdução. Na primeira seção é descrita a metodologia para realizar o diagnóstico; na segunda são apresentados e discutidos os principais achados; e a terceira consiste em considerações finais. 


\section{METODOLOGIA}

O diagnóstico situacional foi parte de um estudo mais amplo do tipo intervenção. O estudo foi desenvolvido em 2018/2019, no âmbito das ações do Núcleo de Estudos em Saúde e Nutrição na Escola (NESANE) junto a escolas públicas de Macaé, Rio de Janeiro. As atividades desenvolvidas foram aprovadas pelo Comitê de Ética em Pesquisa do Hospital Universitário Clementino Fraga Filho (CAAE: 49258313.1.0000.5257). Neste artigo é apresentada apenas a metodologia e os resultados do diagnóstico situacional, primeira etapa do estudo de intervenção.

A metodologia empregada foi descritiva e exploratória. Foram feitas revisão bibliográfica e duas oficinas para discutir a bibliografia analisada e identificar pontos críticos para propor ações de PAAS. Apresentamos a seguir o escopo do NESANE e o detalhamento da metodologia.

O NESANE vincula-se ao Campus UFRJ-Macaé, e desde 2011 realiza atividades de ensino, pesquisa e extensão, incluindo diagnósticos situacionais para desenvolver atividades e materiais educativos de PAAS no âmbito escolar. O conjunto de ações ocorre em diferentes segmentos, abrangendo desde a educação infantil até o ensino médio. As atividades são concebidas no âmbito da extensão universitária, entendida como um processo dinâmico de troca de saberes e interação entre universidade e sociedade (FORPROEX, 2012).

Tendo como norte a diretriz de indissociabilidade da extensão para com a pesquisa e o ensino, o NESANE analisa o perfil alimentar e nutricional de estudantes, professores e funcionários; avalia o ambiente alimentar interno e do entorno das escolas; e identifica a percepção de professores sobre alimentação saudável.

A partir dos resultados dessas avaliações, e pautadas na diretriz extensionista de interação dialógica, são promovidas diferentes ações educativas de PAAS junto à comunidade escolar. Essas incluem oficinas de retorno e discussão de resultados com a comunidade escolar; sessões de vídeodiscussão e cursos para professores sobre nutrição e alimentação saudável; e atividades desenvolvidas de forma curricular com alunos e professores das escolas. Como referência em alimentação saudável, o NESANE se apoia no Guia Alimentar para a População Brasileira - GAPB (BRASIL, 2014); e em termos de educação alimentar e nutricional, no Marco de Referência de Educação Alimentar e Nutricional para Políticas Públicas - MREAN (BRASIL, 2012).

Com vistas a atender a diretriz extensionista de transformação social, o NESANE participa ainda de reuniões de planejamento administrativo junto a profissionais da Secretaria 
Municipal de Educação de Macaé, e à direção das escolas. Além disso, o NESANE participa do planejamento pedagógico junto aos professores das escolas para promover a inserção do tema “alimentação e nutrição" em atividades curriculares. Ressalta-se também o cumprimento da diretriz de fortalecimento da formação universitária, uma vez que graduandos do Campus UFRJ- Macaé participam de todas as atividades mencionadas.

A metodologia do diagnóstico para fins de ações de PAAS foi realizada em três etapas. A primeira etapa consistiu em resgatar publicações do NESANE relacionadas às atividades supracitadas, e sistematizar os principais resultados para permitir uma visão do perfil das escolas. A revisão bibliográfica incluiu apenas as produções do NESANE no período de 2014 a 2019.

A segunda etapa consistiu numa oficina da equipe do NESANE para, à luz do GAPB e do MREAN, analisar as informações sistematizadas (BRASIL, 2014; BRASIL, 2012). A oficina teve duração de quatro horas, e as participantes foram quatro docentes e uma graduanda do último período do Curso de Nutrição do Campus UFRJ-Macaé. Sentadas em círculo, as participantes analisaram a sistematização, que havia sido previamente organizada em apresentação informatizada de slides. Foi dada oportunidade a todas para expressar suas considerações. Uma participante atuou como moderadora das falas, enquanto outra fez o registro dos apontamentos.

Primeiramente o grupo buscou responder à pergunta norteadora: "Quais os principais problemas de alimentação e nutrição nas escolas de Macaé?”. Depois de esgotadas as falas, o grupo passou para a segunda pergunta: “Quais são os entraves no ambiente escolar para a superação desses problemas?”. Ao final, a partir das considerações, o grupo produziu coletivamente um documento com pontos críticos acerca da situação alimentar e nutricional nas escolas. Posteriormente à oficina, o documento foi aprimorado pela relatora e compartilhado individualmente com as participantes para apreciação e ajustes finais.

A terceira etapa foi outra oficina da equipe, que funcionou nos mesmos moldes da primeira, porém com o objetivo de, a partir dos pontos críticos organizados, identificar públicos- alvo e possíveis ações de PAAS que pudessem ser desenvolvidas nas escolas. A pergunta norteadora da discussão foi: “Que ações de PAAS são fundamentais a partir dos pontos críticos?”. Foram pensadas ações de PAAS que, assim como outras atividades de promoção da saúde, podem ocorrer em três dimensões, a saber: incentivo, apoio e proteção. Ações de incentivo visam difundir informação, promover reflexão e encorajar os sujeitos a adotarem práticas 
saudáveis. A dimensão de apoio inclui ações que facilitam a adesão a práticas saudáveis no nível individual e coletivo. Já ações de proteção possuem cunho regulatório e buscam impedir que os indivíduos sejam expostos a ambientes não saudáveis (LOURENÇO; PONTES, 2019a). Após a discussão coletiva, a equipe elaborou um segundo documento com perspectivas de ações de PAAS, o qual foi consolidado pela relatora e enviado para as participantes para apreciação.

\section{RESULTADOS}

Na revisão bibliográfica das publicações do NESANE, foram resgatados quatro relatórios técnicos com informações descritivas das atividades anuais desenvolvidas pelo núcleo, 22 resumos em anais de congressos, três trabalhos de conclusão de curso de graduação (TCC) e um de especialização sobre estado nutricional de escolares (SANTOS, 2014; VIEIRA, 2014; JESUS, 2019; VIGANOR, 2019), um TCC sobre a percepção de professoras de escolas sobre alimentação saudável (MARTINS, 2018), três TCC sobre saúde dos professores das escolas (FREZ, 2019; VIVEIROS, 2019; RODRIGUES, 2019), um artigo completo sobre ambiência e estado nutricional em pré-escolas de Macaé (LOURENÇO et al., 2019), um capítulo de livro sobre diagnóstico nutricional no espaço escolar (LOURENÇO et al., 2018), um livro sobre educação alimentar e nutricional voltado para educadores (CORDEIRO, LOURENÇO; PONTES, 2017), e um livro sobre alimentação na escola, voltado para famílias e educadores (LOURENÇO; PONTES, 2019b).

Ambas as oficinas ocorreram sem entraves e o formato proposto permitiu que todas as participantes se expressassem livremente e, dessa forma, chegassem a um consenso sobre os pontos críticos e as ações deles derivadas. Foram identificados durante a primeira oficina oito pontos críticos acerca da situação alimentar e nutricional nas escolas, os quais se classificaram em cinco temáticas pertinentes à PAAS e encontram-se resumidos no Quadro 1. 
Quadro 1. Pontos críticos acerca da situação alimentar e nutricional em escolas públicas de Macaé- RJ, $2018 / 2019$

\begin{tabular}{|c|c|c|}
\hline \multicolumn{2}{|r|}{$\begin{array}{l}\text { Pontos críticos acerca da situação alimentar e } \\
\text { nutricional nas escolas }\end{array}$} & $\begin{array}{l}\text { Temática no campo da Promoção } \\
\text { da Alimentação Adequada e } \\
\text { Saudável }\end{array}$ \\
\hline 1 & $\begin{array}{l}\text { Elevada prevalência de sobrepeso e obesidade } \\
\text { entre estudantes, professores e funcionários. }\end{array}$ & Perfil Antropométrico \\
\hline 2 & $\begin{array}{l}\text { Presença frequente de alimentos não saudáveis no } \\
\text { lanche escolar }\end{array}$ & Perfil Alimentar \\
\hline 3 & Baixo consumo de água pelos estudantes & $\begin{array}{l}\text { Perfil Alimentar / Ambiente } \\
\text { Alimentar }\end{array}$ \\
\hline 4 & $\begin{array}{l}\text { Presença de cantinas não saudáveis no interior das } \\
\text { escolas }\end{array}$ & Ambiente Alimentar \\
\hline 5 & $\begin{array}{l}\text { Presença de comércio e propaganda de alimentos } \\
\text { não saudáveis no entorno das escolas }\end{array}$ & Ambiente Alimentar \\
\hline 6 & $\begin{array}{l}\text { Lacuna de formação dos professores das escolas } \\
\text { acerca do tema da alimentação saudável }\end{array}$ & Educação Alimentar e Nutricional \\
\hline 7 & $\begin{array}{l}\text { Pais/responsáveis da comunidade escolar } \\
\text { apresentam dúvidas diversas sobre alimentação }\end{array}$ & Educação Alimentar e Nutricional \\
\hline 8 & $\begin{array}{l}\text { Dificuldade de contato com pais/responsáveis e } \\
\text { baixa participação desses em atividades propostas } \\
\text { pela escola }\end{array}$ & Comunicação em Saúde \\
\hline
\end{tabular}

Fonte: Elaboração própria com dados da pesquisa.

Em relação ao perfil nutricional antropométrico, destacou-se como ponto crítico a elevada prevalência de sobrepeso e obesidade entre os estudantes e professores das escolas. Por exemplo, em duas escolas de ensino fundamental-I e duas escolas de ensino médio, a prevalência de excesso de peso (sobrepeso+obesidade) atingiu, respectivamente, 48,0\% e 76,8\% dos professores (FREZ, 2019; RODRIGUES, 2019). Em escolas de educação infantil, a prevalência de excesso de peso foi alarmante entre menores de cinco anos (13,7\%) (LOURENÇO et al., 2019). Perfil semelhante também foi identificado entre adolescentes do ensino fundamental (31,0\%) e médio (19,2\%) (SANTOS, 2014; JESUS, 2019).

No que se refere ao perfil alimentar, observou-se presença frequente de itens 
ultraprocessados no lanche escolar e baixa frequência de frutas ou outros alimentos com processamento industrial reduzido. Por meio da observação direta do lanche trazido por 60 alunos de uma escola de ensino fundamental-I, foi identificada frequência de biscoitos igual a 37\%, biscoitos mais bebidas adoçadas 33\%, além da presença rotineira de outros produtos ultraprocessados, como salgadinhos de pacote e guloseimas (MONTEIRO et al., 2017). Outro ponto crítico foi o baixo consumo de água pelos estudantes. Havia bebedouros nas escolas visitadas, mas, em especial naquelas de ensino fundamental, o uso dos bebedouros não era frequente. Poucos estudantes tinham caneca ou garrafa individual para beber água, e não havia copo descartável disponível. Existia apenas uma caneca única, que permanecia ao lado do bebedouro, para uso comum (LOURENÇO et al., 2017; MARTINS, 2018).

Um aspecto observado acerca do ambiente alimentar foi a presença de cantina não saudável no interior das escolas. Nas escolas de ensino fundamental visitadas pelo NESANE havia cantina comercial, que vendia diariamente pizza de presunto e calabresa; hambúrguer com carne industrializada, catchup e batata palha; refresco industrializado; guaranás sem gás; e guloseimas como balas, pirulitos, chocolates e amendoim colorido. Também havia comércio e propaganda de alimentos não saudáveis no entorno das escolas, tanto no portão de entrada (venda por ambulantes) quanto no raio de três quarteirões (locais comerciais, como bares, padarias e mercearias). Havia propaganda de refrigerantes e salgadinhos de pacote nesses pontos de venda, sendo guaranás sem gás, balas e chicletes alguns dos produtos disponíveis (LOURENÇO et al., 2017; MARTINS, 2018).

Em termos do conhecimento da comunidade escolar sobre alimentação saudável, destacase que a compreensão dos professores sobre o tema diferia daquela proposta no GAPB. Além disso, os professores relataram ter várias dúvidas sobre o assunto, desconhecer referências como o GAPB e o MREAN, e não se sentir preparados para falar sobre alimentação saudável com os alunos (MARTINS, 2018).

Também foi notado um ponto crítico acerca da comunicação entre integrantes da comunidade escolar. Pais/responsáveis também apresentavam dúvidas sobre alimentação saudável e, em linhas gerais, desconheciam o GAPB. Na visão dos professores, era baixa a frequência das famílias nas atividades propostas pela escola. Os professores ressaltaram que essa pouca participação dificultava harmonizar as ações de PAAS feitas nas escolas com as orientações sobre alimentação que os alunos recebiam em casa (MARTINS, 2018). 
A partir dos pontos críticos, foram pensados na segunda oficina objetivos norteadores para ações de PAAS. Considerando as dimensões de incentivo, apoio e proteção, foram também pensados exemplos de ações de PAAS que podem ser desenvolvidas por diferentes atores sociais, envolvendo públicos-alvo variados. Esses exemplos são apresentados no Quadro 2. 
Quadro 2. Perspectivas de ações de Promoção da Alimentação Adequada e Saudável em escolas públicas de Macaé-RJ, 2018/2019

\begin{tabular}{|c|c|c|c|}
\hline \multicolumn{2}{|r|}{ Objetivo da ação } & Dimensão em & Exemplo de ações \\
\hline 1 & \multirow{3}{*}{$\begin{array}{l}\text { Reduzir a prevalência de } \\
\text { sobrepeso e obesidade na } \\
\text { comunidade escolar }\end{array}$} & Incentivo & $\begin{array}{l}\text { Oficinas, cursos, entre outras, para sensibilização de estudantes e famílias sobre estilo de vida ativo e } \\
\text { alimentação saudável. }\end{array}$ \\
\hline & & Apoio & Criação de feiras e hortas nas escolas e arredores. \\
\hline & & Proteção & Ações que estimulem o cumprimento das leis sobre comercialização de alimentos no espaço escolar. \\
\hline \multirow[t]{3}{*}{2} & \multirow{3}{*}{$\begin{array}{l}\text { Aprimorar a qualidade do } \\
\text { lanche trazido pelos } \\
\text { estudantes }\end{array}$} & Incentivo & Produção e distribuição para estudantes e famílias de materiais educativos sobre lanches saudáveis. \\
\hline & & Apoio & Adequação de horários e refeitórios para favorecer a adesão pelos estudantes às refeições do PNAE. \\
\hline & & Proteção & Regulamentação dos alimentos que podem ser trazidos pelos estudantes para a escola. \\
\hline & \multirow{3}{*}{$\begin{array}{l}\text { Favorecer o consumo de } \\
\text { água pelos estudantes }\end{array}$} & Incentivo & Atividades de cunho artístico ou cultural que motivem o consumo de água pelos estudantes. \\
\hline & & Apoio & Disponibilizar nas escolas bebedouros com água filtrada e utensílio adequado para o consumo. \\
\hline & & Proteção & Análise da qualidade da água ofertada na escola para garantir conformidade com padrões legais. \\
\hline \multirow[t]{3}{*}{4} & \multirow{3}{*}{$\begin{array}{l}\text { Retirar cantinas não } \\
\text { saudáveis do interior das } \\
\text { escolas }\end{array}$} & Incentivo & Atividades educativas para sensibilização da direção escolar e cantineiros. \\
\hline & & Apoio & Transformar cantinas em cantinas saudáveis. \\
\hline & & Proteção & Ações de fiscalização quanto ao cumprimento das leis vigentes acerca das cantinas escolares. \\
\hline \multirow[t]{3}{*}{5} & \multirow{3}{*}{$\begin{array}{l}\text { Qualificar o ambiente } \\
\text { alimentar no entorno das } \\
\text { escolas }\end{array}$} & Incentivo & Ações de sensibilização da comunidade sobre a importância de qualificar o entorno das escolas. \\
\hline & & Apoio & Ofertar alimentação adequada e saudável no espaço escolar. \\
\hline & & Proteção & Não permitir propagandas e comercialização de alimentos não saudáveis no portão das escolas. \\
\hline \multirow[t]{3}{*}{6} & \multirow{3}{*}{$\begin{array}{l}\text { Qualificar professores } \\
\text { acerca do tema da } \\
\text { alimentação saudável }\end{array}$} & Incentivo & Cursos de formação para professores sobre alimentação saudável. \\
\hline & & Apoio & Disponibilizar horário para os professores realizarem cursos de formação. \\
\hline & & Proteção & Garantir o cumprimento das leis sobre inserção do tema alimentação e nutrição no currículo escolar. \\
\hline \multirow[t]{3}{*}{7} & \multirow{3}{*}{$\begin{array}{l}\text { Orientar pais/responsáveis } \\
\text { sobre } \\
\text { saudável }\end{array}$} & Incentivo & Atividades educativas que estimulem a reflexão das famílias sobre alimentação saudável. \\
\hline & & Apoio & Disponibilizar para as famílias materiais educativos sobre alimentação saudável. \\
\hline & & Proteção & Ações para esclarecer as famílias sobre as leis acerca da alimentação no ambiente escolar. \\
\hline \multirow[t]{3}{*}{8} & \multirow{3}{*}{$\begin{array}{l}\text { Estreitar o diálogo entre a } \\
\text { escola e as famílias da } \\
\text { comunidade escolar }\end{array}$} & Incentivo & Construção de materiais educativos em linguagem acessível, para divulgação em diferentes mídias. \\
\hline & & Apoio & Disponibilizar na escola acesso à internet para estudantes e famílias. \\
\hline & & Proteção & Assegurar leis trabalhistas sobre condições dignas de jornada e sobrecarga. \\
\hline
\end{tabular}


Produzir e distribuir para estudantes e suas famílias materiais educativos sobre lanches saudáveis ou sobre a importância de beber água foram exemplos de ações de PAAS sugeridas pelo diagnóstico na dimensão de incentivo, ou seja, que buscam informar e sensibilizar a comunidade escolar sobre alimentação saudável. Já na dimensão de apoio, transformar cantinas escolares em cantinas saudáveis e disponibilizar bebedouros com água filtrada foram duas dentre outras possibilidades de ações levantadas, que podem dar condições práticas para que os estudantes tenham hábitos alimentares saudáveis. No que se refere à dimensão de proteção, o diagnóstico traz como exemplo ações que envolvem regras coletivas ou leis que visam proteger os estudantes e todos da comunidade escolar contra a exposição a alimentos não saudáveis, como a regulamentação do que pode ser comercializado nas cantinas escolares, e a proibição de propagandas de alimentos não saudáveis no entorno das escolas.

São sem dúvida diversos os desafios para implementar ações de PAAS nas escolas. É essencial considerar que existem diferentes camadas em que se pode atuar, dependendo da posição social que se ocupa. Profissionais da educação, até mesmo pela natureza de sua formação, têm amplo potencial para promover ações na dimensão de incentivo, tais como cursos, oficinas e rodas de conversa com estudantes e famílias. Porém, essas também podem ser desenvolvidas por profissionais da rede municipal de saúde, ou pela universidade em parceria com as escolas no contexto da extensão universitária. Tais parcerias intersetoriais são um caminho promissor, pois dividem a responsabilidade das ações ao mesmo tempo que encorajam o engajamento social em prol de um objetivo comum. Destaca-se que as ações de PAAS na dimensão de incentivo são francamente encorajadas pelo MREAN (BRASIL, 2012), e que o GAPB fornece os subsídios teóricos adequados e atualizados para a elaboração das atividades educativas (BRASIL, 2014).

Já ações nas dimensões de apoio e proteção estão frequentemente no nível de governabilidade da direção escolar ou dos gestores municipais. Mas é pertinente que professores, estudantes e famílias estejam cientes dessas possibilidades para que possam cobrar e apoiar a execução das ações. Por exemplo, ações de proteção relacionadas à fiscalização do cumprimento de leis que regulamentam a comercialização de alimentos nas escolas devem ser de conhecimento de todos da comunidade escolar, ainda que apenas alguns tenham a gerência principal. Cabe comentar que tais ações encontram-se legalmente respaldadas nas esferas federal e estadual (BRASIL, 2006; BRASIL, 2007, RIO DE JANEIRO, 2014). 
É preciso ter em mente que para a escola ser promotora de alimentação adequada e saudável é necessário que o conjunto de atores envolvidos trabalhe de maneira articulada (LOURENÇO; PONTES, 2019a; POLICY BRIEF, 2021). Porém, também é necessário reconhecer que essa articulação de pessoas, organizações e ideias é um dos maiores desafios no âmbito das ações sociais e de saúde coletiva. Sendo assim, ressalta-se a importância do diagnóstico aqui apresentado como uma forma de motivar e orientar movimentos individuais e coletivos de PAAS.

O atual cenário epidemiológico nas escolas brasileiras inclui elevada prevalência de excesso de peso entre estudantes e professores, e é frequente a presença de alimentos não saudáveis (IBGE, 2016; LOURENÇO; PONTES, 2019b). Além disso, são aspectos facilitadores de ações de PAAS o fato da Educação Alimentar e Nutricional encontrar-se incorporada por lei no currículo escolar (BRASIL, 2018), e da sociedade estar buscando conformidade com as regulamentações sobre comercialização de alimentos no ambiente escolar (BRASIL, 2007). As escolas encontram-se, portanto, perméáveis para a implementação de ações de PAAS na contemporaneidade.

\section{CONSIDERAÇÕES FINAIS}

O resultado mais importante deste estudo é a própria geração de um diagnóstico que pode direcionar variadas ações de PAAS no âmbito escolar. A partir desse material, diferentes atores sociais podem, dentro de seu nível de gerência, desenvolver de forma assertiva políticas e ações nas três dimensões de PAAS. Essa classificação em dimensões não visa delimitar o foco das ações de maneira estrita, mas auxiliar cada ator a visualizar o nível de gerência e as possibilidades de alcance das ações.

As publicações sistematizadas deram conta de gerar um panorama da situação alimentar e nutricional em escolas de Macaé. Isso ressalta o potencial do conhecimento científico oriundo da extensão universitária. Tais publicações relataram informações coletadas de maneira continuada, em diferentes escolas municipais, por universitários e profissionais locais, e poderão ser utilizadas para orientar ações no próprio município. Fica em destaque, portanto, a relevância do Campus UFRJ-Macaé e da extensão universitária para a sociedade macaense (SILVA et al., 2020; LOURENÇO; BERGOLD, 2019).

O fato deste diagnóstico possibilitar que as ações sejam contextualizadas com a realidade das escolas de Macaé é um ponto forte deste estudo, mas limita a generalização de 
seus resultados. Entretanto se acredita que os pontos críticos identificados podem ser comuns a escolas de outras localidades brasileiras e, portanto, trazer aprendizados para outros municípios.

O vínculo academia-comunidade resultante da extensão universitária, como ocorre no NESANE, favorece intervenções contextualizadas e com maior potencial transformador. Destaca-se a escola como cenário para a prática extensionista e PAAS, por ser um espaço de formação de hábitos e troca de saberes. Ao apontar pontos críticos em nível local, os diagnósticos situacionais agregam concretude aos problemas, promovem reflexão entre os envolvidos e, consequentemente, contribuem também para a materialização das ações e a transformação social. Este estudo abre um leque de ações de PAAS que podem ser desenvolvidas no espaço escolar de Macaé. Nesse sentido é vasto o potencial de desenvolvimento local a partir de um diagnóstico como o aqui produzido.

\section{REFERÊNCIAS}

BRASIL. Decreto de lei no 13.666, de 16 de maio de 2018 (Altera a Lei 9394/1996). Diário Oficial da União - Seção 1 - 17/05/2018.

BRASIL. Fundo Nacional de Desenvolvimento da Educação (FNDE). Portaria Interministerial $\mathrm{n}^{\circ} 1.010$ de 08 de maio de 2006 . Institui as diretrizes para a Promoção da Alimentação Saudável nas Escolas de educação infantil, fundamental e nível médio das redes públicas e privadas, em âmbito nacional, 2006.

BRASIL. Ministério da Educação. Secretaria de Educação Superior (SESu). A democratização e expansão da educação superior no país 2003-2014. Brasília: SESu; 2015. BRASIL. Ministério da Saúde, Secretaria de Atenção à Saúde, Departamento de Atenção Básica. Cadernos de Atenção Básica - Saúde na Escola. Brasília: Ministério da Saúde, 2009.

BRASIL. Ministério da Saúde. Plano de ações estratégicas para o enfrentamento das doenças crônicas não transmissíveis (DCNT) no Brasil 2011-2022. Brasília: Ministério da Saúde, 2011. Disponível em:

https://bvsms.saude.gov.br/bvs/publicacoes/plano_acoes_enfrent_dent_2011.pdf .

BRASIL. Ministério da Saúde. Regulamentação da Comercialização de Alimentos em Escolas no Brasil: Experiências estaduais e municipais. Brasília, 2007. Disponível em: http://nutricao.saude.gov.br/pas.php?conteudo=publicacoes_pas

BRASIL. Ministério da Saúde. Secretaria de Atenção à Saúde. Departamento de Atenção Básica. Guia alimentar para a população brasileira / Ministério da Saúde, Secretaria de Atenção à Saúde, Departamento de Atenção Básica, v.2, Brasília: Ministério da Saúde, 2014. 
BRASIL. Ministério do Desenvolvimento Social e Combate à fome. Marco de Referência de Educação Alimentar e Nutricional para as Políticas Públicas - Brasília, DF: Secretaria Nacional de Segurança Alimentar e Nutricional, 2012.

CORDEIRO, A. A.; LOURENÇO, A. E. P.; PONTES, P. V. Brincar, comer, nutrir, atividades lúdicas para a Educação Infantil. Curitiba: Editora CRV, 2017.

FORPROEX. Fórum de Pró - Reitores de Extensão Universitária das Instituições públicas de Educação Superiores Brasileiras. Política Nacional de Extensão Universitária. Porto Alegre, RS: UFRGS, 2012.

FREZ, J. S. Consumo alimentar e Síndrome de Burnout em professores do ensino fundamental I da rede pública de Macaé. Trabalho de Conclusão de Curso de Graduação em Nutrição na Universidade Federal do Rio de Janeiro, Campus UFRJ Macaé Professor Aloísio Teixeira, 2019.

IBGE. INSTITUTO BRASILEIRO DE GEOGRAFIA E ESTATÍSTICA. Pesquisa de Orçamentos Familiares 2017-2018. Análise do consumo alimentar pessoal no Brasil. Rio de Janeiro: Instituto Brasileiro de Geografia e Estatística, 2020.

IBGE. INSTITUTO BRASILEIRO DE GEOGRAFIA E ESTATÍSTICA. Pesquisa Nacional de Saúde do Escolar 2015. Rio de Janeiro: Instituto Brasileiro de Geografia e Estatística, 2016.

JESUS, I. N. H. Perfil nutricional antropométrico de adolescentes de escolas públicas de Macaé, Rio de Janeiro. Trabalho de Conclusão de Curso de Graduação em Nutrição na Universidade Federal do Rio de Janeiro, Campus UFRJ Macaé Professor Aloísio Teixeira, 2019.

LOURENÇO, A. E. P.; BERGOLD, L. B. (orgs). Saberes e experiências de extensão em promoção da saúde. Campos dos Goytacazes, RJ: Essentia, 2019.

LOURENÇO, A. E. P.; PONTES, P. V. Ambiente alimentar e promoção da alimentação saudável na escola. In: LOURENÇO, A. E. P.; PONTES, P. V. Conversa sobre Alimentação na Escola. Curitiba: Editora CRV, 2019, p.99-107.

LOURENÇO, A. E. P.; PONTES, P. V. Conversa sobre Alimentação na Escola. Curitiba: Editora CRV, 2019.

LOURENÇO, A. E. P.; PONTES, P. V.; PINTO, T. J. P.; MONTEIRO, L. S.; SPERANDIO, N.; ROCHA, C. M. M.; LINS, L. R.; GABRIEL, R. C. F. Percepção de professoras sobre o ambiente alimentar na escola. Anais do I Seminário de Ambiente Alimentar. ENSP/FIOCRUZ, 2017.

LOURENÇO, A. E. P.; ROCHA, C. M. M.; PINTO, T. J. P. Avaliação nutricional na escola: a pesquisa direcionando ações de saúde e nutrição. In: ALMEIDA, M. F. L.; CAPELLI, J. C. S.; SPERANDIO, N.; ROCHA, C. M. M.; RIBEIRO, B. G. Alimentação e Nutrição da Infância à Adolescência: Diálogo Multidisciplinar com a Prática em Saúde. São Paulo, Editora Triall Editora Ltda, 2018. 
LOURENÇO, A. E. P.; VIEIRA, J. L.; ROCHA C. M. M.; LIMA, F. F. Influência da ambiência escolar no estado nutricional de pré-escolares de Macaé. Ciência \& Saúde e Coletiva, vol.24, n.7, 2019. p. 2399-2410

MARTINS, V. S. Percepção de professoras sobre alimentação saudável em uma escola pública de ensino fundamental I de Macaé. Trabalho de Conclusão de Curso de Graduação em Nutrição na Universidade Federal do Rio de Janeiro, Campus UFRJ Macaé Professor Aloísio Teixeira, 2018.

MONTEIRO, L. S.; PINTO, T. J. P.; SPERANDIO, N.; PONTES, P. V.; FERREIRA, L. C. C.; FREZ, J. S.; LACERDA, M. G.; LOURENÇO, A. E. P. Consumo alimentar e associação com perfil antropométrico de adolescentes de Macaé/RJ. In: Anais do Congresso Brasileiro de Epidemiologia, 2017. Anais eletrônicos: Campinas, Galoá, 2017. Disponível em: https://proceedings.science/epi/trabalhos/consumo-alimentar-e-associacao-com-perfilantropometrico-de-adolescentes-de-macaerj

POLICY BRIEF. Obesidade Infantil. estratégias para prevenção e cuidado (em nível local). Disponível em: $<$ https://centrodeexcelencia.org.br/wp-content/uploads/2021/05/Policy- BriefObesidade-pt.pdf $>$. Acesso em: 17 jul 2021.

RIO DE JANEIRO (Estado). Lei no 6859 de 07 de julho 2014. Disponível em: https://leisestaduais.com.br/rj/lei-ordinaria-n-6859-2014-rio-de-janeiro-dispoe-sobreorganizacao-e-o-funcionamento-das-cantinas-escolares-na-rede-publica-de-ensino-do-estadodo-rio-de-janeiro.

RODRIGUES, W. T. O. Educação e Saúde: Relação entre o trabalho e a saúde de professores do ensino estadual de Macaé, Rio de Janeiro. Trabalho de Conclusão de Curso de Graduação em Nutrição na Universidade Federal do Rio de Janeiro, Campus UFRJ Macaé Professor Aloísio Teixeira, 2019.

SANTOS, T. C. dos. Avaliação nutricional de adolescentes de uma escola pública do município de Macaé, Rio de Janeiro. Trabalho de Conclusão de Curso de Graduação em Nutrição na Universidade Federal do Rio de Janeiro, Campus UFRJ Macaé Professor Aloísio Teixeira, 2014.

SILVA, G. A. B. E; SILVA, S. R. DE A. E; SOUZA, I. L. DE; MONTEIRO, L. S. Parceria entre governo local e universidades: formação do Observatório da Cidade de Macaé no contexto de rede colaborativa interinstitucional e intersetorial. Vértices. v.22, n.3, 2020. p.412-428

SILVA, S. R. A.; CARVAlHO, M. R. Macaé, do Caos ao conhecimento: olhares acadêmicos sobre o cenário de crise econômica. Macaé: Prefeitura Municipal de Macaé, 2019.576p.

VIEIRA, J. L. Estado nutricional de crianças entre 24- 59 meses e a ambiência de escolas municipais de educação infantil de Macaé, RJ. Trabalho de Conclusão de Curso de Graduação em Nutrição na Universidade Federal do Rio de Janeiro, Campus UFRJ Macaé Professor Aloísio Teixeira, 2014. 
VIGANOR, J. T. Utilização da Razão Cintura/Estatura na avaliação nutricional de adolescentes. Trabalho de Conclusão de Curso de Especialização em Nutrição Clínica na Universidade Federal do Rio de Janeiro, 2019.

VIVEIROS, L. C. F. Qualidade de Vida e Síndrome de Burnout em Professores de Escolas da Rede de Ensino do Município de Macaé-RJ. Trabalho de Conclusão de Curso de Graduação em Nutrição na Universidade Federal do Rio de Janeiro, Campus UFRJ Macaé Professor Aloísio Teixeira, 2019.

WORLD HEALTH ORGANIZATION. Global strategy on diet, physical activity and health, Geneva: WHO, 2004. $\quad$ Disponível em; https://www.who.int/dietphysicalactivity/strategy/eb11344/strategy_english_web.pdf 